Research Paper

\title{
GABAergic Alterations in the Rat Testis after Methamphetamine Exposure
}

\author{
Paweena Kaewman ${ }^{1,2}$, Sutisa Nudmamud-Thanoi ${ }^{1,2}$, Samur Thanoi ${ }^{1,2}{ }^{\llbracket}$ \\ 1. Department of Anatomy, Faculty of Medical Science, Naresuan University, Phitsanulok 65000, Thailand. \\ 2. Centre of Excellence in Medical Biotechnology, Faculty of Medical Science, Naresuan University, Phitsanulok 65000, Thailand. \\ $\square$ Corresponding author: Tel: +66 55 964600; Fax: +66 55 964770; E-mail: samurt@nu.ac.th \\ (c) Ivyspring International Publisher. This is an open access article distributed under the terms of the Creative Commons Attribution (CC BY-NC) license \\ (https:// creativecommons.org/licenses/by-nc/4.0/). See http://ivyspring.com/terms for full terms and conditions.
}

Received: 2018.06.01; Accepted: 2018.07.29; Published: 2018.08.10

\begin{abstract}
Gamma-aminobutyric acid (GABA), GABA-A receptors and GABA transporter 1 (GAT1) were reported to be involved in the proliferation of Leydig cells, testosterone production and spermatogenesis. Since methamphetamine (METH) has been reported to have adverse effects on testis and its functions, the aim of this study was therefore to determine the changes of GABAergic activity in testis after METH exposure. Male Sprague-Dawley rats were divided into control, acute binge (AB-METH), escalating dose (ED METH) and escalating dose-binge (ED-binge METH) groups. After sacrifice, rat testes were removed and used to estimate GABA concentration and the expression of GABA-A receptor, GAD1, GAD2 and GAT1 genes by using HPLC and RT-PCR, respectively. The GABA concentration was significantly increased in all METH-administrated groups. In addition, significant increases of GABA-A $\alpha 1$ receptor and GADI genes expression were found in the ED-binge METH group. Gene expressions of GATI were numerically decreased in all METH-administrated rats and reached significant in the ED METH group. These results indicated a compensatory upregulation of GABA production and its functions in testis after METH exposure. Thus, these changes might represent a homeostatic response of GABAergic to the adverse effects of METH.
\end{abstract}

Key words: Rat testis, Methamphetamine, GABA, GABA-A a1 receptor, Glutamate decarboxylase, GAT1.

\section{Introduction}

Indicators of GABAergic neuronal function include gamma-aminobutyric acid (GABA), GABA receptors, GABA transporter (GAT) and glutamate decarboxylase (GAD); the GABA synthesizing enzymes. The expression of such GABAergic markers has been reported not only in the brain but also in peripheral tissues including the testis. Several subunits of the GABA receptors were found in rodent and human testes while the GABA synthesizing enzymes were also detected: GAD67 and GAD65, which are encoded by GAD1 and GAD2 genes respectively. Interestingly, the GABA-A a1 receptor, GAD67 and GAD65 were found localized to Leydig cells [1]. These findings indicate that the synthesis of GABA might occur in testis. In the testis, GABA acts through its receptors, especially the GABA-A receptors. Changes in GAD and GABA receptors can influence GABA concentration and synaptic activity, respectively. Whereas, a decrease of GAT1 can result in an increase in GABA concentration. The activity of GABA via GABA-A receptors is involved in the proliferation of Leydig cells and testosterone production [2,3]. Additionally, GABA can act as an inhibitory regulator of spermatogenesis. The enhancement of GABA activity can suppress the proliferation of spermatogonial stem cells (SSCs) [4]. Moreover, GAT1 influences the expression and the role of testicular function [5]. Abnormalities of Leydig cells including small size and decreased number were found in transgenic mice with abnormal GAT1 expression [6]. These findings show that GABAergic activity plays an important role in controlling testicular functions.

Methamphetamine (METH) abuse can cause addiction and adverse effects on the nervous and male reproductive systems. Several studies have 
reported impairments of testis, sperm quality and endocrine disruption after METH exposure [7-10]. Apoptosis of Leydig cells and a decrease of serum testosterone were found [8, 9]. Furthermore, METH administration resulted in the reduction of progesterone and estrogen receptor expression in testis [9]. This evidence indicates the powerful adverse effect of METH on testicular function. Interestingly, the changes of sperm quality and the expression of androgen receptor in Leydig cells was found in a rat model of addiction after the administration of GABA and a GABA-containing food supplement [11]. Thus, this study proposed to investigate the alterations of GABAergic measures in the testis of METH-administered rats.

\section{Materials and methods}

\section{Animals and METH administration}

Male Sprague-Dawley rats were housed and maintained at $24 \pm 1^{\circ} \mathrm{C}$ under 12 hours of the light/dark cycle with free access to water and food. METH administration protocol was approved by Naresuan University Animal Care and Use Committee, Thailand. The protocols were adapted from Segal et al. [12]. Briefly, the rats were divided into 4 groups ( $\mathrm{n}=8-10$ per group) including control, acute binge (AB METH), escalating dose (ED METH) and escalating-binge METH (ED-binge METH) groups. The rats in the control group were injected intraperitoneally (i.p.) with $0.9 \%$ saline for 15 days. In the AB METH group, rats were injected (i.p.) with saline for 14 days followed by METH binge dose, 6 $\mathrm{mg} / \mathrm{kg}$ METH in 4 times a day at $2 \mathrm{~h}$ intervals, on day 15 . The rats in the ED METH group were treated in parallel with in the ED-binge METH group. They were injected (i.p.) with a gradually increasing dose of METH $0.1-4 \mathrm{mg} / \mathrm{kg}$, for 14 days and either $0.9 \%$ saline or METH binge dose on day 15 for the ED METH and ED-binge METH groups, respectively. At the end of the treatment, testes were removed immediately after sacrifice and kept at $-80^{\circ} \mathrm{C}$.

\section{High performance liquid chromatography (HPLC) analysis}

Testis was thawed and homogenized on ice with deionized water. After that the homogenate was centrifuged at $10,000 \mathrm{rpm}$ for 20 minutes at $4{ }^{\circ} \mathrm{C}$. The supernatant was collected, then precipitated with $50 \%$ methanol. Following precipitation, the sample was centrifuged again at $10,000 \mathrm{rpm}$ for 20 minutes at $4{ }^{\circ} \mathrm{C}$. Supernatant was collected and filtered with a $0.22 \mu \mathrm{m}$ polyvinylindene fluoride membrane. The GABA concentration in the rat testis was analyzed using HPLC with electrochemical detection. O-Phthaldialdehyde (OPA)/Sulfite was used as derivatizing reagent. It was added into filtrated supernatant. Then, the supernatant was injected onto a C18 reverse phase (pore size $5 \mu \mathrm{m}$ ) column, with a mobile phase consisting of $0.1 \mathrm{M}$ Sodium Phosphate in $8 \%$ methanol (HPLC grade) $\mathrm{pH}$ 5.84. Electrochemical conditions were $+700 \mathrm{mV}$ for guard cell potential, $+400 \mathrm{mV}$ for E1 and $+650 \mathrm{mV}$ for E2. A flow rate of $1 \mathrm{ml} / \mathrm{min}$ was used for peak separation. Column temperature and auto-sample injection chamber was controlled at $25^{\circ} \mathrm{C}$ and $4{ }^{\circ} \mathrm{C}$ respectively. This method was validated with chromatographic parameters validation tests including precision, linearity and accuracy tests [13]. The peak areas of samples were recorded and the GABA concentration calculated.

\section{Reverse transcription polymerase chain reaction (RT-PCR) technique}

Total RNA was isolated from testis using the TRIzol ${ }^{\mathrm{TM}}$ Reagent (Invitrogen, Carlsbad, CA) and chloroform. Then, it was used to synthesize complementary DNA (cDNA) using a qScript ${ }^{\mathrm{TM}}$ XLT cDNA Supermix (Quanta Biosciences, Gaithersburg, $\mathrm{MD})$ according to the manufacturer's protocol. Oligonucleotide primers of GABAergic system genes are show in Table 1. The primer for GABA-A a1 receptor was used according to a previous study [14]. All Other primers were designed by using Primer3 software (freely available at http://bioinfo.ut.ee/ primer3-0.4.0/) and Oligo Analyzer software version 1.0.3 (Freeware, Teemu Kuulasmaa, Finland). The RT-PCR sequences were amplified using a PerfeCTa SYBR Green FastMix, ROX (Quanta Biosciences, Gaithersburg, MD) and performed on a PCRmax Eco 48 real-time PCR system (PCRmax, Staffordshire, UK). Then, the gene expression levels were represented as relative mRNA expression values of interested genes that were normalized with reference gene (GAPDH gene).

Table 1. Sequence of oligonucleotide primers for gene expression in rat testis using RT-PCR

\begin{tabular}{lll}
\hline Genes & Forward primer $\left(5^{\prime} \mathbf{3}^{\prime}\right)$ & Reverse primer $\left(5^{\prime} \mathbf{3}^{\prime}\right)$ \\
\hline GABA-A a1 & TGTCTTTGGAGTGACGACCGTTCT & ACACGAAGGCATAGCACACTGCAA \\
GAD1 (GAD67) & CCTGGAGCTGGCTGAATACC & TGTGCTCAGGCTCACCATTG \\
GAD2 (GAD65) & GATCGGAACAGACAGCGTGAT & GCACTCACCAGGAAAGGAACA \\
GAT1 & GCAGATGACACCACTCACCA & ATGACCTGGAGACGCTGCTT \\
GAPDH & AGTGCCAGCCTCGTCTCATA & GACTGTGCCGTTGAACTTGC \\
\hline
\end{tabular}




\section{Statistical analysis}

The data are shown as mean \pm SEM. The differences between groups were analyzed by using One-way ANOVA followed by Dunnett's post hoc test. Statistical significance was considered at $\mathrm{p} \leq 0.05$.

\section{Results}

\section{GABA concentration in rat testis}

The results of the relative standard deviation of intraday and interday precision were $0.24 \%$ and $1.925 \%$, respectively. The linearity test and accuracy test were acceptable at $\mathrm{R}^{2}=0.9999$ and recovery rate $=$ $100.1 \%$. The concentrations of GABA were significantly increased in AB-METH, ED-METH and
ED-binge METH groups compared to a control group $(p \leq 0.05)$ (Figure 1).

\section{GABA-A $\alpha 1$ receptor, GAD1, GAD2 and GAT1 genes expression in rat testis}

The mRNA expressions of GABA-A a1 receptor and GAD1 gene were significantly increased in ED-binge METH group compared to the control group (Figure 2 and Figure 3). However, gene expression of GAD2 was not changed in all METH-administrated groups (Figure 4), while, a significant decrease of GAT1 was found in the ED METH group compared to the control group (Figure $5)$.

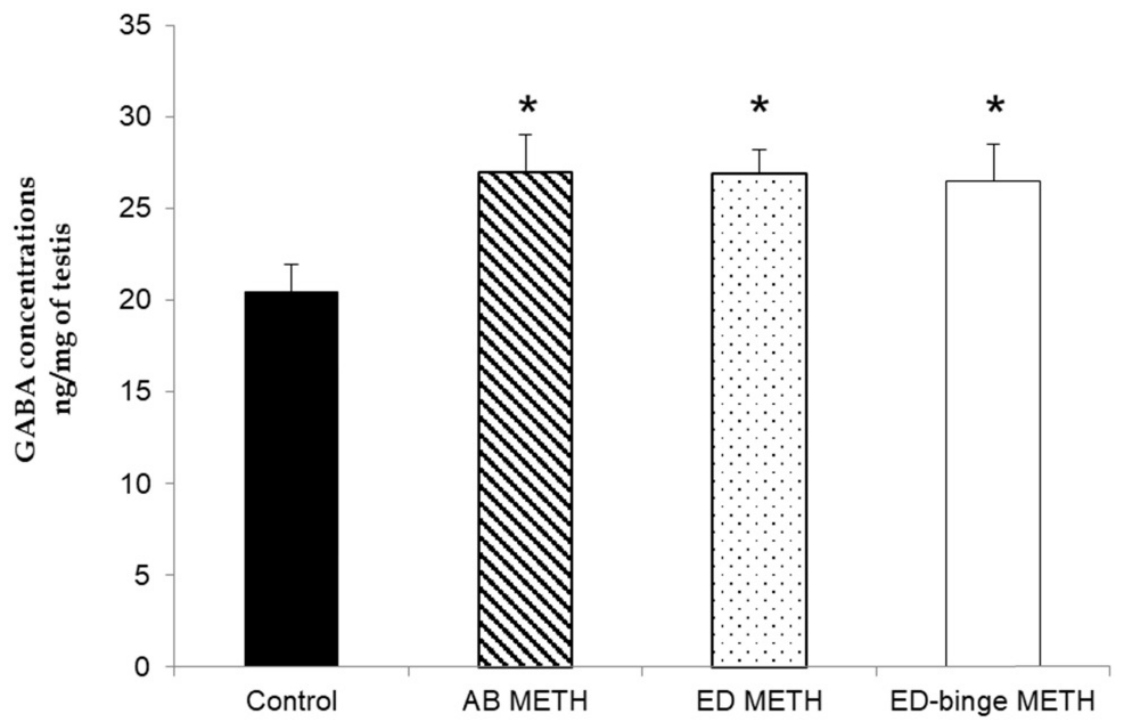

Figure 1. GABA concentration in rat testis of METH-administration groups compared to a control group. Values are mean \pm SEM, $n=8-10$ per group $(* p \leq 0.05$; Dunnett's post hoc test).

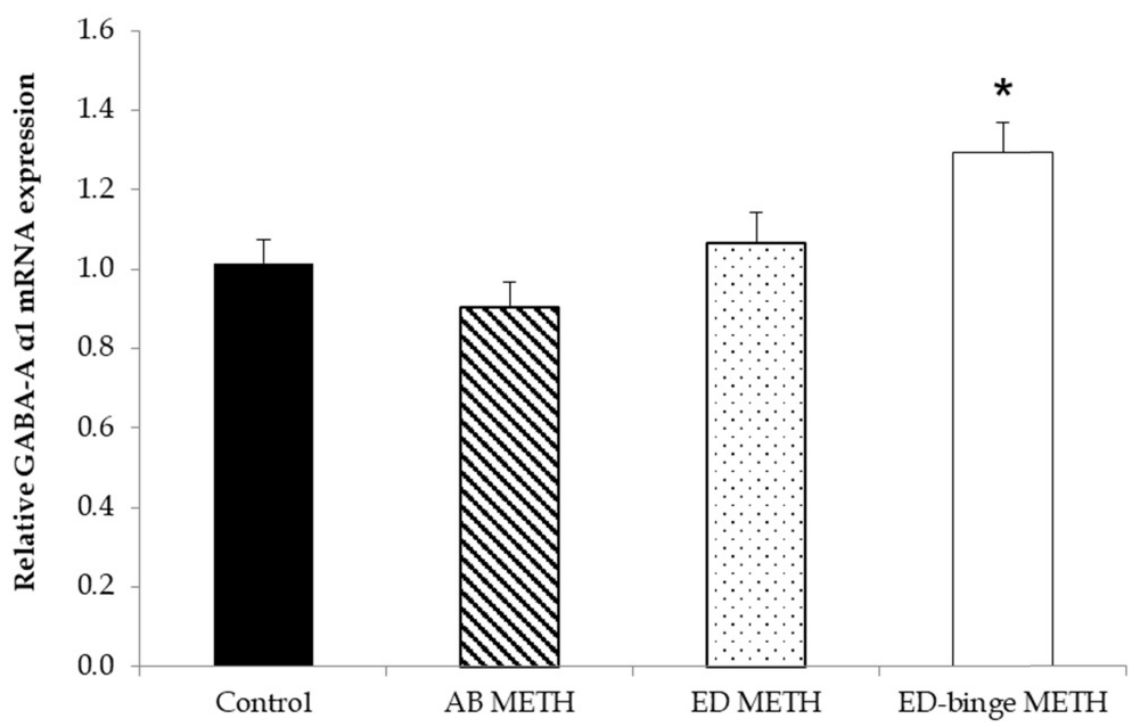

Figure 2. The relative GABA-A $\alpha 1$ receptor $m R N A$ expression in rat testis after METH administrations compared to a control group. Values are mean \pm SEM, $n=9-10$ per group (*P $\leq 0.05$; Dunnett's post hoc test). 


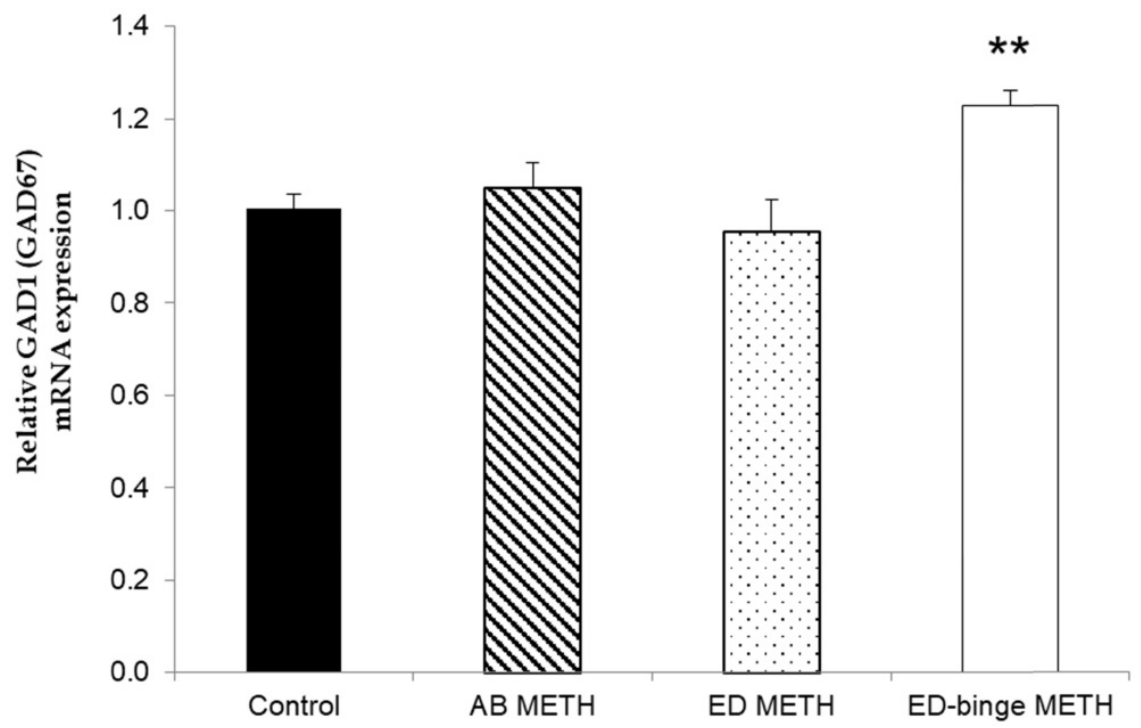

Figure 3. The relative GADI (GAD67) mRNA expression in rat testis after METH administrations compared to a control group. Values are mean \pm SEM, $n=9-10$ per group $(* * \mathrm{p}<0.01$; Dunnett's post hoc test).

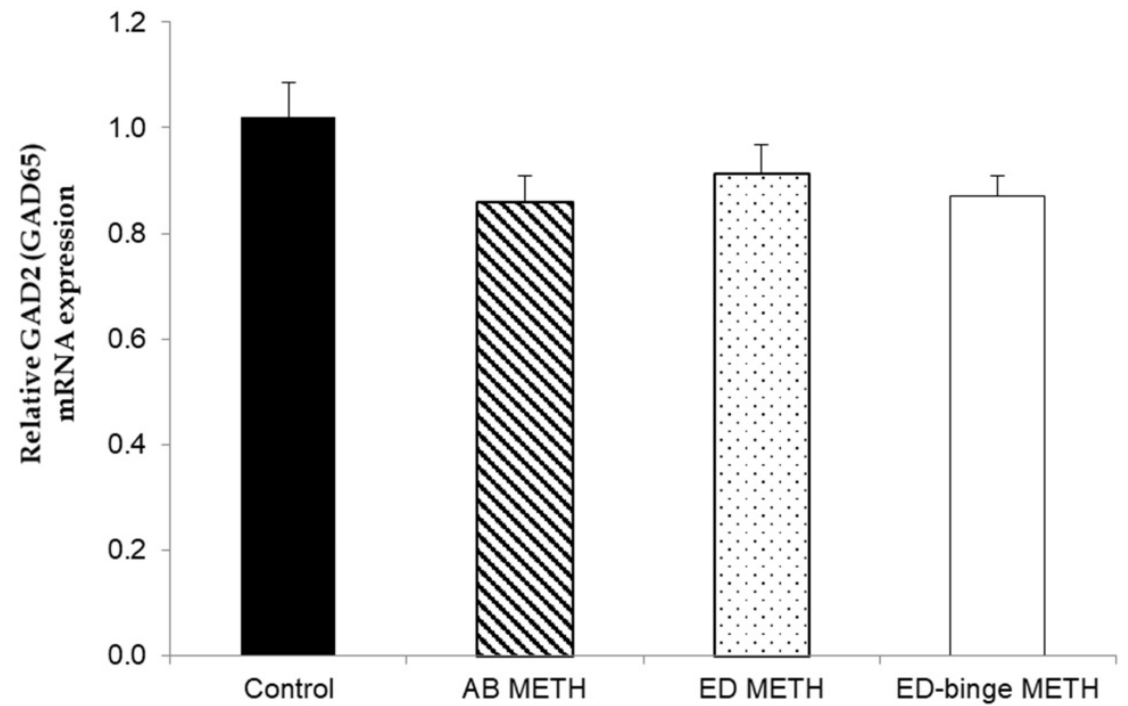

Figure 4. The relative GAD2 (GAD65) mRNA expression in rat testis after METH administrations compared to a control group. Values are mean \pm SEM, $n=9-10$ per group.

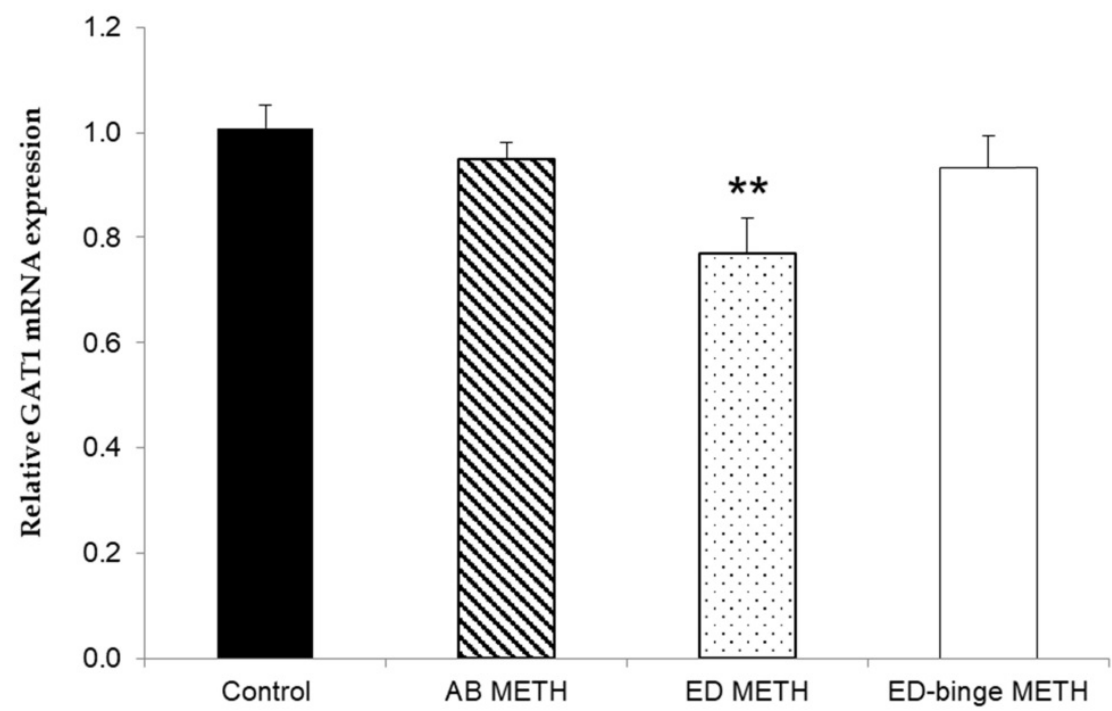

Figure 5. The relative GAT1 mRNA expression in rat testis after METH administrations compared to a control group. Values are mean \pm SEM, $n=9-10$ per group $(* * p<0.01$; Dunnett's post hoc test). 


\section{Discussion}

Our findings showed that METH administration can bring about an increase of GABA concentration. In parallel with this change, gene expression of GABA-A a1 receptor and GABA synthesizing enzyme, GAD1, in the testis were increased after METH administration. Additionally, a reduction of the GAT1 gene expression in METH-administrated rats indicated that GABA reuptake was inhibited. The reduction of GABA reuptake resulted in an enhancement of the GABA concentration [15]. Therefore, the results of this study demonstrated the stimulation of GABA production and function in the testis in response to the adverse effect of METH exposure.

METH exposure has been reported to induce apoptosis of spermatogenic cells as well as damage to Leydig cells and their functions [8, 9]. Since Leydig cell proliferation [3] and the production of testosterone [2] are regulated by the GABAergic activity, the increases of GABA concentration and GABA-A a1 receptor in the testis of METH-administrated rats might be compensatory responses to the effects of METH. Moreover, the inhibitory effect of GABA can inhibit SSC proliferation, the initial spermatogenic cells in spermatogenesis [4]. Thus, high activity of GABA in response to METH may result in spermatogenesis arrest in the testis. Additionally, there was a report that the abnormal expression of GAT1 in the testis leads to the impairment of spermatogenesis and testosterone production [6]. The present study, therefore, also provides further indications of the imbalance of the GABAergic function in the testis that might enhance testicular abnormalities.

In this study, changes in the GAD1 gene but not in the GAD2 gene could reflect that GAD1 gene is more responsive to the adverse effect of METH than GAD2 gene in the testis. There was a previous study showing that the activity of GAD in Leydig cells was found only in GAD67 protein, encoded by the GAD1 gene [3]. Similarly, our results also demonstrated that METH influenced GAD1 gene that specifically acts on Leydig cells.

Interestingly, the ED-binge METH administration which is actually mimic the use of METH in METH abusers can enhance abnormalities of sperm morphology [10]. Furthermore, our previous study showed that the concentration of norepinephrine and its metabolite in testis were attenuated in the ED-binge METH group [16]. Consistent with these findings, we found that not only GABA concentration but also the expression of GAD1 and GABA-A a1 receptor genes were obviously changed in the ED-binge METH group. The association of the inhibitory regulation of GABA with norepinephrine release was reported in another peripheral tissue such as the adrenal gland. GABA activation by the GABA-A receptor agonists caused the inhibition of the norepinephrine release from the adrenal gland [17]. With the similar regulation in the adrenal gland, an increase of GABA activity in the testis in response to METH might inhibit the release of norepinephrine which may cause spermatogenesis arrest [18].

This study focused on the determination of GABAergic gene expression because there are relatively few studies supporting the role of GABAergic in the testis and the mechanism of the GABAergic changes is still unclear. Our results demonstrated the changes of GABAergic in METH-administered rats. These could reflect that GABAergic activity might be a useful therapeutic target to improve reproductive dysfunction caused by METH exposure.

In conclusion, the present study supports the important role of GABA in the testis. GABA concentration, GAD1 and GABA-A receptor gene expressions were increased while GAT1 gene expression was decreased in testis of METH-administrated rats. These changes could reflect the increase of GABA concentration and its activity in an attempt to compensate the adverse effects of METH on male reproductive function.

\section{Abbreviations}

GABA: gamma-aminobutyric acid; GAT: GABA transporter; GAD: glutamate decarboxylase; METH: Methamphetamine; $\mathrm{AB}$ : acute binge; ED: escalating dose; HPLC: High performance liquid chromatography; RT-PCR: Reverse transcription polymerase chain reaction.

\section{Acknowledgements}

This study was supported by Thailand Research Fund (TRF) and Naresuan University Research Fund. PK was supported by the Royal Golden Jubilee Ph.D. Program, Thailand. We appreciate the facilities support from Naresuan University.

\section{Competing Interests}

The authors have declared that no competing interest exists.

\section{References}

1. Geigerseder C, Doepner R, Thalhammer A, Frungieri MB, Gamel-Didelon K, Calandra RS, et al. Evidence for a GABAergic system in rodent and human testis: local GABA production and GABA receptors. Neuroendocrinology. 2003; 77: 314-23.

2. Taherianfard M, Bahaddini A, Keshtkar S, Fazeli M, Shomali T. Effect of Extremely Low Frequency Electromagnetic Field and GABAA Receptors on Serum Testosterone Level of Male Rats. Int J Endocrinol Metab. 2013; 11: e11029. 
3. Geigerseder C, Doepner RF, Thalhammer A, Krieger A, Mayerhofer A. Stimulation of TM3 Leydig cell proliferation via GABA(A) receptors: a new role for testicular GABA. Reprod Biol Endocrinol. 2004; 2: 13.

4. Du Y, Du Z, Zheng H, Wang D, Li S, Yan Y, et al. GABA exists as a negative regulator of cell proliferation in spermatogonial stem cells. [corrected]. Cell Mol Biol Lett. 2013; 18: 149-62.

5. Zhang J, Gui Y, Yuan T, Bian C, Guo L. Expression of GAT1 in male reproductive system and its effects on reproduction in mice. Syst Biol Reprod Med. 2009; 55: 175-80.

6. $\mathrm{Hu} \mathrm{JH}$, Zhang JF, Ma YH, Jiang J, Yang N, Li XB, et al. Impaired reproduction in transgenic mice overexpressing Gamma-aminobutyric acid transporter I (GAT1). Cell Res. 2004; 14: 54-9.

7. Yamamoto Y, Yamamoto K, Hayase T, Abiru H, Shiota K, Mori C. Methamphetamine induces apoptosis in seminiferous tubules in male mice testis. Toxicol Appl Pharmacol. 2002; 178: 155-60.

8. Nudmamud-Thanoi S, Thanoi S. Methamphetamine induces abnormal sperm morphology, low sperm concentration and apoptosis in the testis of male rats. Andrologia. 2011; 43: 278-82

9. Lin JF, Lin YH, Liao PC, Lin YC, Tsai TF, Chou KY, et al. Induction of testicular damage by daily methamphetamine administration in rats. Chin J Physiol. 2014; 57: 19-30.

10. Nudmamud-Thanoi S, Sueudom W, Tangsrisakda N, Thanoi S. Changes of sperm quality and hormone receptors in the rat testis after exposure to methamphetamine. Drug Chem Toxicol. 2016; 39: 432-8.

11. Thanoi S, Roboon J, Nudmamud-Thanoi S. Recovery effect of pre-germinated brown rice on the changes of sperm quality, testicular structure and androgen receptor expression in a rat model of drug addiction. Int J Med Sci 2018; 15(9): 921-928.

12. Segal DS, Kuczenski R, O'Neil ML, Melega WP, Cho AK. Escalating dose methamphetamine pretreatment alters the behavioral and neurochemical profiles associated with exposure to a high-dose methamphetamine binge. Neuropsychopharmacology. 2003; 28: 1730-40.

13. Monge-Acuna AA, Fornaguera-Trias J. A high performance liquid chromatography method with electrochemical detection of gamma-aminobutyric acid, glutamate and glutamine in rat brain homogenates. J Neurosci Methods. 2009; 183: 176-81.

14. Wearne TA, Parker LM, Franklin JL, Goodchild AK, Cornish JL. GABAergic mRNA expression is differentially expressed across the prelimbic and orbitofrontal cortices of rats sensitized to methamphetamine: Relevance to psychosis. Neuropharmacology. 2016; 111: 107-18.

15. Soudijn W, van Wijngaarden I. The GABA transporter and its inhibitors. Curr Med Chem. 2000; 7: 1063-79.

16. Janphet S, Nudmamud-Thanoi S, Thanoi S. Alteration of catecholamine concentrations in rat testis after methamphetamine exposure. Andrologia. 2017; 49: 11.

17. Kataoka Y, Fujimoto M, Alho H, Guidotti A, Geffard M, Kelly GD, et al. Intrinsic gamma aminobutyric acid receptors modulate the release of catecholamine from canine adrenal gland in situ. J Pharmacol Exp Ther. 1986; 239: 584-90.

18. Mhaouty-Kodja S, Lozach A, Habert R, Tanneux M, Guigon C, Brailly-Tabard $\mathrm{S}$, et al. Fertility and spermatogenesis are altered in \{alpha\} $1 \mathrm{~b}$-adrenergic receptor knockout male mice. J Endocrinol. 2007; 195: 281-92. 